\title{
Numerical simulation of polymer film stretching
}

\author{
Hogenrich Damanik ${ }^{1}$, Abderrahim Ouazzi ${ }^{1}$, and Stefan Turek ${ }^{1}$ \\ Institut für Angewandte Mathematik, TU Dortmund, Vogelpothsweg 87 \\ hdamanik@mathematik.tu-dortmund.de
}

\begin{abstract}
We present numerical simulations of a film stretching process between two rolls of different temperature and rotational velocity. Film stretching is part of the industrial production of sheet of plastics which takes place after the extrusion process. The goal of the stretching of the sheet material is to rearrange the orientation of the polymer chains. Thus, the final products have more smooth surfaces and homogeneous properties. In numerical simulation, the plastic sheet is being modelled geometrically as a membrane and rheologically as a polymer melt. The thickness of the membrane is not assumed to be constant but rather depends on the rheology of the polymer and heat transfer. The rheology of the sheet material is governed by a viscoelastic fluid and is coupled to the flow model. An A-stable time integrator is applied to the systems in which the continuous spatial system is discretized within the FEM framework at each time step. The resulting discrete systems are solved via Newton-multigrid techniques. Here, a level set method is used to capture the free surfaces. We obtain similar results for test configurations with available results from literature and present "neck-in" as well as "dog-bone" effects.
\end{abstract}

\section{Introduction}

Film casting processes are widely practiced in industry. The purpose is to produce thin sheets of polymer. They are mainly used for food packaging, drugs, coating, etc. Having extruded from the die below the melting temperature, the film sheet needs to be further oriented on the molecular level to obtain more smooth material properties at desired thickness. This is done by several rolls stretching the sheet material, see Fig. 1. The first several rolls warm up the temperature of the sheet material with constant heat source. Then, the two middle rolls stretch the sheet with different velocity and temperature. The last several rolls cool down the temperature of the sheet material. Industrial objectives are to improve the properties of end products at higher production rates and to reduce production cost. In reality, the higher the rate of production process is the poorer the quality of the end product becomes. The reduced qualities are well-knwon as "neck-in" and "dog-bone/edge-bead" effects as studied in [8] for the extrusion process.

Numerical treatment of such process has been studied in the work of [7] where a $2 \mathrm{D}$ membrane model is introduced together with isothermal Newtonian flow. Furthermore, a viscoelastic model is clearly of importance to the corresponding process, as shown in $[13,14]$. 


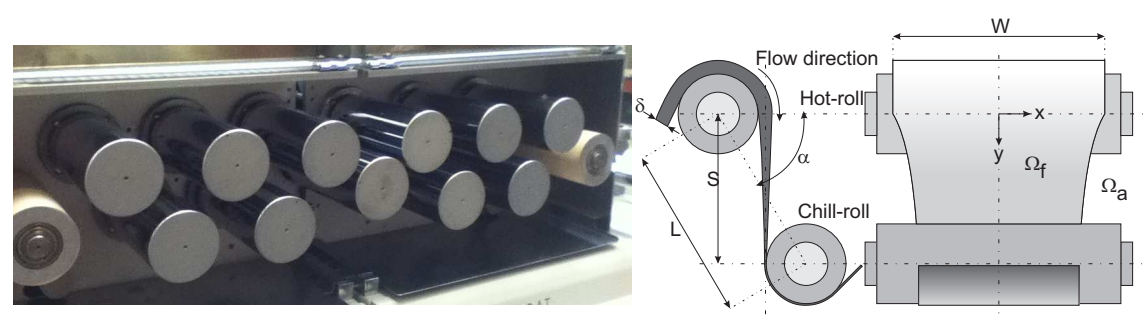

Fig. 1. Left: Laboratory tools (Courtesy of Schöpfner, Wibbeke). Right: The numerical setup

\section{Membrane model, viscoelasticity and temperature coupling}

The thickness of the sheet material film, which is roughly $0.1 \%$ of the width, makes it possible to use a $2 \mathrm{D}$ membrane model of [7]. This is numerically more advantageous, but we are aware of physical limitations of using this model which is that one cannot predict the whole physical phenomena, in particular when dealing with viscoelastic constitutive laws, see [10]. Following [7], the steady state 2D model starts with the assumption that inertia can be neglected and that incompressibility holds, such as

$$
\nabla \cdot e \mathbf{T}+\rho \mathbf{g} e=0 \text { and } \nabla \cdot e \mathbf{u}=0 \text { where } \mathbf{T}=-p \mathbf{I}+2 \eta \mathbf{D}
$$

for Newtonian fluids. The thickness $e$ appears as new unknown inside the equation. This is a consequence of the $2 \mathrm{D}$ simplification of the third direction in $3 \mathrm{D}$. Further consequence is that the pressure equals to the $2 \mathrm{D}$ velocity gradient

$$
p=-2 \eta \operatorname{tr}(\mathbf{D}) \text { with } \mathbf{D}=\frac{1}{2}\left(\nabla \mathbf{u}+\nabla \mathbf{u}^{T}\right)
$$

So, equation (1) can be written without pressure as unknown, respectively only velocity and thickness $(\mathbf{u}, e)$ as unknowns

$$
\nabla \cdot e(2 \eta \mathbf{D}+2 \eta \operatorname{tr}(\mathbf{D}) \mathbf{I})+\rho \mathbf{g} e=0 \text { and } \nabla \cdot e \mathbf{u}=0 .
$$

In the case of nonstationary problems, equation (3) becomes

$$
\frac{\partial \mathbf{u}}{\partial t}+\nabla \cdot e(2 \eta \mathbf{D}+2 \eta \operatorname{tr}(\mathbf{D}) \mathbf{I})+\rho \mathbf{g} e=0 \text { and } \frac{\partial e}{\partial t}+\nabla \cdot e \mathbf{u}=0
$$

In the presence of viscoelasticity, the total stress becomes $\mathbf{T}=-p \mathbf{I}+2 \eta_{s} \mathbf{D}+$ $\frac{\eta_{p}}{\Lambda}(\boldsymbol{\tau}-\mathbf{I})$, where the conformation stress tensor $\boldsymbol{\tau}$ is governed by the following viscoelastic constitutive law,

$$
\frac{\partial \boldsymbol{\tau}}{\partial t}+(\mathbf{u} \cdot \nabla) \boldsymbol{\tau}-\nabla \mathbf{u} \cdot \boldsymbol{\tau}-\boldsymbol{\tau} \cdot \nabla \mathbf{u}^{T}=\frac{1}{\Lambda} f(\boldsymbol{\tau}),
$$


with parameters $\eta_{s}, \eta_{p}, \Lambda$ as solvent viscosity, polymer viscosity and polymer relaxation time. Depending on how one sets $f(\boldsymbol{\tau})$, many viscoelastic models such as in [9] can be included. In this study, we use the Oldroyd-B model with $59 \%$ solvent contribution, if later not explicitely mentioned. Furthermore, since the non-isothermal condition should be treated, a transport of temperature is available via the following equation

$$
\frac{\partial \theta}{\partial t}+(\mathbf{u} \cdot \nabla) \theta=k_{1} \nabla^{2} \theta
$$

which influences the viscosity and the relaxation time of the fluid, as for example by the well-known Arrhenius dependence

$$
\eta_{\theta}=\eta_{0} \exp \frac{E}{R}\left(\frac{1}{\theta}-\frac{1}{\theta_{0}}\right), \quad \Lambda_{\theta}=\Lambda \exp \frac{E}{R}\left(\frac{1}{\theta}-\frac{1}{\theta_{0}}\right) .
$$

Here, the parameters $k_{1}, E, R$ are the heat diffusion coefficient, activation of energy and ideal gas constant.

\section{Multiphase treatment}

In the presence of the free surface for the above setup configuration, a surface tracking method is possible. However, it is more convenient to use a single mesh without one has to update the mesh at every time step. Furthermore, an ALE formulation makes also sense since the deformation of the mesh is small. On the other hand, it is computationally cheaper, in view of nonisothermal situations, to avoid additional numerical variables exerting from ALE formulation. Thus, a level set equation [12] is a good candidate,

$$
\frac{\partial \varphi}{\partial t}+(\mathbf{u} \cdot \nabla) \varphi=0
$$

to capture the free surface as recently implemented in [6] for multiphase viscoelastic flow. One needs to take care that the function should approximate the distance property of $\|\nabla \varphi\|=1$ at each time step, if not should satisfy.

\section{Numerical treatment}

The numerical strategy to deal with the multiphase character, that means where and what to solve, is based on the sign of the level set function. In the following we describe the numerical treatment via the backward Euler scheme, for simplicity. We proceed, also implemented in $[3,4]$ and by neglecting the gravity (also for simplicity), as follows: Given initial solutions $\left(\mathbf{u}^{n}, e^{n}, \boldsymbol{\tau}^{n}, \theta^{n}\right)$ and interface $\varphi^{n}$ in each time step, the fully coupled weak formulation of the 
above system of equations is to find $\left(\mathbf{u}=\mathbf{u}^{n+1}, e=e^{n+1}, \boldsymbol{\tau}=\boldsymbol{\tau}^{n+1}, \theta=\right.$ $\left.\theta^{n+1}\right)$ for the next time step with $\Delta t=t^{n+1}-t^{n}$ so that

$$
\begin{gathered}
\frac{1}{\Delta t}\langle\mathbf{u}, \phi\rangle_{\Omega}-\langle e 2 \eta(\mathbf{D}+\operatorname{tr}(\mathbf{D}) \mathbf{I}), \nabla \phi\rangle_{\Omega}+\langle(e \mathbf{T}) \cdot \mathbf{n}, \phi\rangle_{\partial \Omega}=\frac{1}{\Delta t}\left\langle\mathbf{u}^{n}, \phi\right\rangle_{\Omega} \\
\frac{1}{\Delta t}\langle e, \phi\rangle_{\Omega}+\langle(e \nabla \cdot \mathbf{u}+(\mathbf{u} \cdot \nabla) e), \phi\rangle_{\Omega}=\frac{1}{\Delta t}\left\langle e^{n}, \phi\right\rangle_{\Omega} \\
\frac{1}{\Delta t}\langle\boldsymbol{\tau}, \phi\rangle_{\Omega}+\left\langle(\mathbf{u} \cdot \nabla) \boldsymbol{\tau}-\nabla \mathbf{u} \cdot \boldsymbol{\tau}-\boldsymbol{\tau} \cdot \nabla \mathbf{u}^{T}-\frac{1}{\Lambda} f(\boldsymbol{\tau}), \phi\right\rangle_{\Omega}=\frac{1}{\Delta t}\left\langle\boldsymbol{\tau}^{n}, \phi\right\rangle_{\Omega} \\
\frac{1}{\Delta t}\langle\theta, \phi\rangle_{\Omega}+\langle(\mathbf{u} \cdot \nabla) \theta, \phi\rangle_{\Omega}+k_{1}\langle\nabla \theta, \phi\rangle_{\Omega}=\frac{1}{\Delta t}\left\langle\theta^{n}, \phi\right\rangle_{\Omega}
\end{gathered}
$$

with an admissible inner product $\langle\cdot, \cdot\rangle$, and with test functions $\phi \in Q_{2}$ as higher order finite element functions. Then, given a current solutions $\mathbf{u}$, one seeks a solution for $\varphi$ of the next time step via

$$
\frac{1}{\Delta t}\langle\varphi, \phi\rangle_{\Omega}+\langle(\mathbf{u} \cdot \nabla) \varphi, \phi\rangle_{\Omega}=\frac{1}{\Delta t}\left\langle\varphi^{n}, \phi\right\rangle_{\Omega}
$$

also here with $\phi \in Q_{2}$ as higher order finite element approximation. The numerical parameters are set to be level set dependent, denoting $\Omega_{f}$ and $\Omega_{a}$, where $\Omega=\Omega_{f} \cup \Omega_{a}$ with $\Omega_{f} \cap \Omega_{a}=0$. Next, a redistancing procedure maintains the distanced property of the level set function in each time step [6]. The process is then repeated for the next time steps.

One expects that the solution may not be smooth even at lower Weissenberg number [11]. This fact introduces problems with the Galerkin formulation. Unlike in the Stokes problem where a pair of $Q_{2} P_{1}$ FEM satisfies the so-called LBB condition for velocity-pressure [1], in the presence of viscoelasticity, Bonito and Burman [2] have shown that there is a second inf-sup stability condition as well for the approximation of velocity-stress. A remedy can be obtained by adding a consistent stabilization term penalizing the jump of the solution gradient over element edges $\mathrm{E}$ (with $h_{E}$ denoting the length of the edge). As presented in [15], this jump term "smooths" also spurious velocity components, thus avoiding unnecessary numerical artifacts. This term can be written in the following form (see [15] for more details and also [4] in the case of viscoelasticity):

$$
J_{\sigma}=\sum_{\text {edge } \mathrm{E}} \gamma h_{E}^{2} \int_{E}[\nabla \boldsymbol{\tau}]:[\nabla \phi] d s
$$

Regarding the numerical solvers, the obtained discrete system of equations (9-14) is nonlinear and fully coupled. Therefore, a damped Newton interation is applied to the solution vector

$$
\mathbf{x}^{n+1}=\mathbf{x}^{n}+\omega^{n}\left[\frac{\partial \mathcal{R}\left(\mathbf{x}^{n}\right)}{\partial \mathbf{x}}\right]^{-1} \mathcal{R}\left(\mathbf{x}^{n}\right),
$$

where $\mathbf{x}$ represents the vector of the coefficients corresponding to the above physical unknowns, with a damping parameter $\omega^{n}$. The resulting linear system are solved via a monolithic multigrid solver, see [5]. 


\section{$5 \quad$ Numerical results}

There exist several numerical attempts for similar problems of film casting, as for example in $[7,13,14]$. Unfortunately there is no common benchmark on this issue. Numerical parameters of the corresponding fluids model are not easily available, thus new numerical techniques are hard to validate. So, here we try the dimensionless numbers of numerical attempts from the following Tab. 1, which use the same geometry as the one in the work of [14] with similar fluid parameters. Comparison with reference is still possible qualitatively, as shown in Fig. 2. We simulate several mesh levels $(12 \times 8,24 \times 16,48 \times 32$, denoted as

Table 1. Film stretching condition

\begin{tabular}{lccccccccc}
\hline Case & Dist.(S) & $(1 / 2 \mathrm{~W})$ & Thick. $(\delta)$ & $u_{\text {in }}$ & $u_{\text {out }}$ & $\theta_{\text {in }}$ & $\theta_{\text {out }}$ & $\eta_{0}$ & We \\
Newtonian & 5 & 12.5 & 0.07 & 0.1 & 1.5 & - & - & 1 & 0 \\
Viscoelastic & 5 & 12.5 & 0.07 & 0.1 & 1.5 & - & - & 1 & 0.03 \\
\hline
\end{tabular}

L2, L3, L4 accordingly) to be sure that the solutions are converging. The following Fig. 2 shows that our numerical results lead to converged solutions with mesh refinement. The end width of the free surface is wider than that of [14]. As a consequence, the thickness along the symmetry line is thinner than that of [14] accordingly. In general, the results show a similar behaviour when the fluid is stretched. Here, the "dog-bone" effect is clearly visible from the left of Fig. 2 In the presence of viscoelasticity, see Fig. 3, the shear thinning
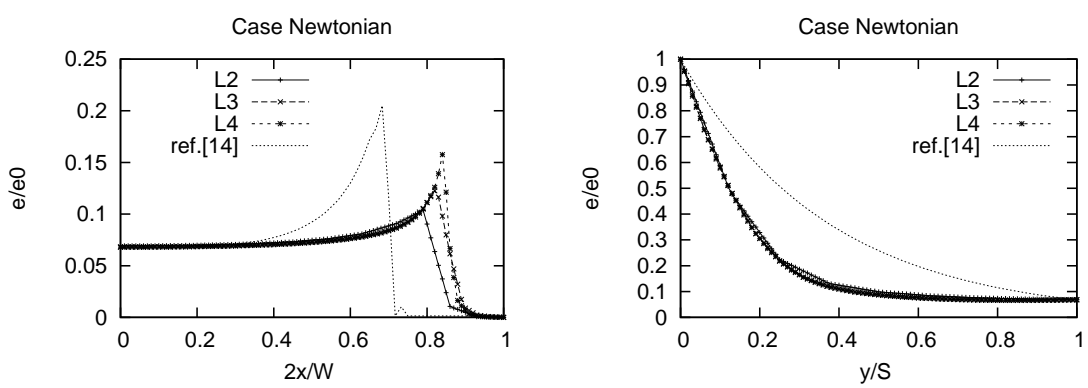

Fig. 2. Thickness profile at the chill roll and the symmetry line

effect in the direction of the elongational flow makes the end width of the film to be wider than that in the case of Newtonian which is also qualitatively shown in [14] with the Upper Convective Maxwell model. As in the Newtonian 

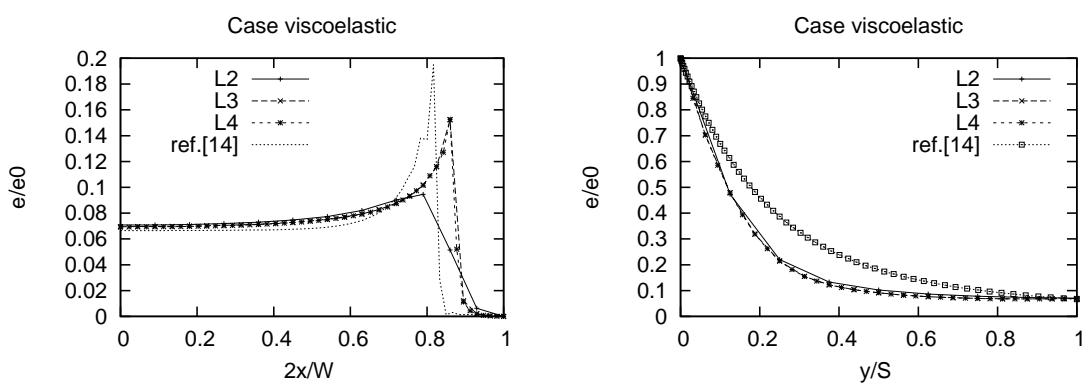

Fig. 3. Thickness profile at the chill roll and the symmetry line

case, one sees clearly "neck-in" effects for the corresponding numerical setup.

Having qualitatively compared the numerical results, we do simulations for the setup in Fig. 1 with the following conditions in Tab. 2. The geometry and flow condition is slightly adjusted from the one in Tab. 1. Here, the temperature of the two rolls is taken into account. The rest of the data is served only for numerical tests. For this setup, we are quite flexible to choose the time step size $(\Delta t=0.01$ is also used in Tab. 1$)$ due to the monolithic treatment of velocity, thickness and stress. Care has to be taken that the

Table 2. Film stretching condition of Fig. 1

\begin{tabular}{lccccccccccc}
\hline Dist.(S) & $(1 / 2 \mathrm{~W})$ & Thick. $(\delta)$ & $u_{\text {in }}$ & $u_{\text {out }}$ & $\theta_{\text {in }}$ & $\theta_{\text {out }}$ & $\eta_{0}$ & We & $\mathrm{E}$ & $\mathrm{R}$ & $k_{1}$ \\
3.487 & 7 & 0.02 & $5 / 3$ & 5 & 433 & 413 & 1 & 0.04779 & 45 & 8.31 & 0 \\
\hline
\end{tabular}

time step size is not too big, not to disturbe the decoupling of the level set. We found that the above time step size is quite optimal in this case. One sees in Fig. 4 that the flow of the system gets steady. It is shown by the thickness evolution of two points: one in the middle of the exit, and one is close to the edge of the free surface. For two levels of computation, the solutions seem to reach mesh convergence. In the region close to free sueface, care needs to be taken that numerical parameters do not give additional "jumps" across the element otherwise the resolution must be high enough to capture smooth datas. However this is not the case in the region $\left(\Omega_{f}\right)$ far from the free surface which shows clearly mesh converged solutions, see Fig. 5. Furthermore, the simulation of the complete system shows the same behaviour of "dog-bone" effect. Here, the thickness in the middle is relatively thinner than that near the free surface. 

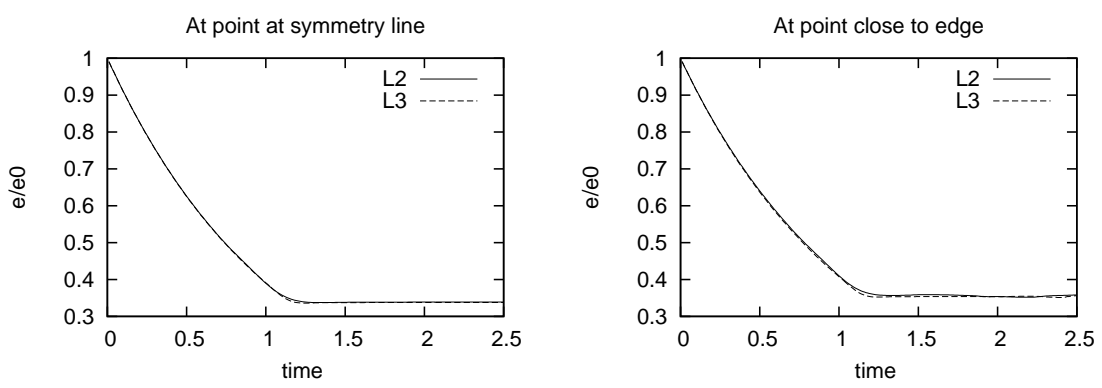

Fig. 4. Thickness evolution at two points at the chill roll (exit/take up)
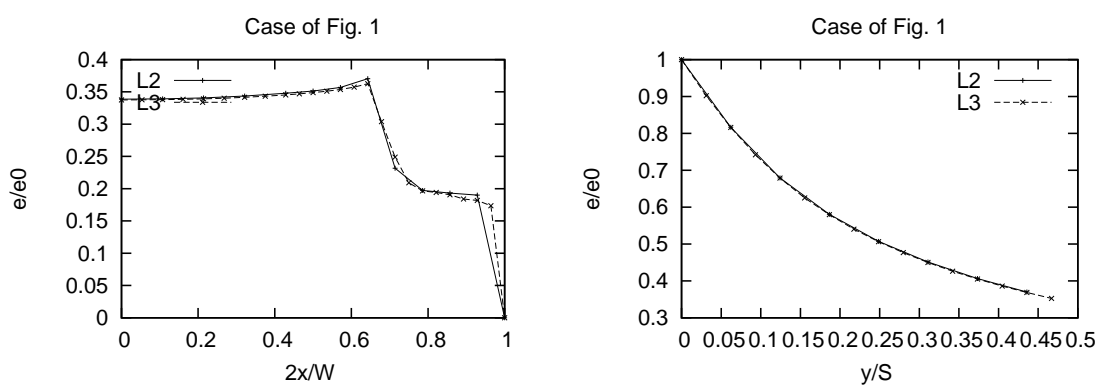

Fig. 5. Thickness profile at the chill roll (exit/take up)

\section{Summary}

A membrane model for simulating the stretching of viscoelastic flow is presented. The total governing system includes the Stokes equations as well as viscoelastic constitutive laws and they are fully coupled. The multiphase characteristic is handled by a level set equation denoting where and what to solve. This may induce numerical artifacts which is then remedied by adding a consistent jump stabilization term. The results are qualitatively compared against reference [14] results and show that the numerical simulation is able to predict the so-called "dog-bone" and "neck-in" effects. In the presence of viscoelasticity, the final thickness at the chill roll shows less of these effects. Further studies will be performed.

\section{Acknowledgement}

The authors would like to thank the German Reasearch Foundation (DFG) for supporting the work through collaborative research center SFB/TR TRR 30 (TPC3) and BMBF-Project 05M13RDC. 


\section{References}

1. Arnold, D.N., Boffi, D., AND FAlK, R.S., Approximation by Quadrilateral Finite Elements, Math. Comput. 71:239 (2002), 909-922.

2. Bonito, A. And Burman, E., A continuous interior penalty method for viscoelastic flows, Siam Journal of Scientific Computing 30 (2008), 1156-1177.

3. Damanik, H., Hron, J., Ouazzi, A., And Turek, S., A monolithic FEMmultigrid solver for non-isothermal incompressible flow on general meshes, Journal of Computational Physics 228 (2009), 3869-3881.

4. __ A monolithic FEM approach for the log-conformation reformulation (lcr) of viscoelastic flow problems, Journal of non-Newtonian Fluid Mechanics 165 (2010), 1105-1113.

5. __ Monolithic newton-multigrid solution techniques for incompressible nonlinear flow models, International Journal for Numerical Methods in Fluids 71 (2012), 208-222.

6. Damanik, H., Ouazzi, A., And Turek, S., Numerical simulation of a rising bubble in viscoelastic fluids, Numerical Mathematics and Advanced Applications Enumath 2011, Springer, Berlin, 2011, ISBN 978-3-642-33133-6, pp. 489497.

7. D'Halewyn, S., Demay, Y., And Agassant, J. F., Numerical simulation of the cast film process, Polymer Engineering and Science 30 (1990), 335-340.

8. Dobroth, T. And Erwin, L., Causes of edge beads in cast films, Polymer Engineering and Science 26 (1986), 462-467.

9. Joseph, D. D., Fluid Dynamics of Viscoelastic Liquids, Springer, 1990, Applied Mathematical Sciences 84.

10. Keunings, R., An algorithm for the simulation of transient viscoelastic flows with free surfaces, J. Computational Physics 62 (1986), 199-220.

11. Renardy, M., A comment on smoothness of viscoelastic stresses, Journal of Non-Newtonian Fluid Mechanics 138 (2006), 204-205.

12. Sethian, J., Level Set Methods and Fast Marching Methods: Evolving Interfaces in Computational Geometry, Fluid Mechanics, Computer Vision, and Material Science, Cambridge University Press, 1999, 2nd edition.

13. Silagy, D., Demay, Y., And Agassant, J. F., Stationary and stability analysis of the film casting process, J. Non-Newtonian Fluid Mech. 79 (1998), 563583.

14. Sollogoub, C., Demay, Y., And Agassant, J. F., Non-isothermal viscoelastic numerical model of the cast-film process, J. Non-Newtonian Fluid Mech. 138 (2006), 76-86.

15. TuREK, S. AND OuAzZI, A., Unified edge-oriented stabilization of nonconforming FEM for incompressible flow problems: Numerical investigations, J. Numer. Math. 15 (2007), 299-322. 